\title{
Studies on the Performance of Some Silkworm, Bombyx mori L, Breeds in Temperate Region of Jammu and Kashmir, India
}

\author{
Bharath Kumar Neelaboina*, Shivkumar, Mir Nisar Ahmad and M.K. Ghosh \\ Central Sericultural Research and Training Institute, Central Silk Board, Galandar, \\ Pampore-192121, Jammu and Kashmir, India \\ *Corresponding author
}

\begin{tabular}{|l|}
\hline K e y w o r d s \\
$\begin{array}{l}\text { Silkworm, Parental } \\
\text { breeds, Temperate, } \\
\text { Evaluation index }\end{array}$ \\
\hline Article Info \\
\hline $\begin{array}{l}\text { Accepted: } \\
15 \text { October } 2018 \\
\text { Available Online: } \\
10 \text { November } 2018\end{array}$ \\
\hline
\end{tabular}

\section{A B S T R A C T}

The present investigation was carried out at Central Sericultural Research and Training Institute, Pampore, Jammu \& Kashmir (J\&K) during spring, summer and autumn, 2017 to study the performance of eleven silkworm, Bombyx mori L, breeds (CSR2, CSR6, CSR27, CSR26, CSR50, PAM114, PAM117, APS4, APS5, SK6 and SK7) of different origin under temperate climatic conditions. The rearing was carried out under uniformed laboratory condition by adopting standard method. These eleven breeds were evaluated for their performance in eight metric traits viz., fecundity (No.), hatching (\%), larval weight $(\mathrm{g})$, yield per 10,000 larvae by number and by weight $(\mathrm{kg})$, single cocoon weight $(\mathrm{g})$, single shell weight $(\mathrm{g})$ and shell ratio $(\%)$. The performance of breeds varied from season to season since they were originated from different progenitors. The data generated was analyzed statistically and subjected to multiple trait Evaluation index. On the basis of the evaluation index values ranking (Mano et al., 1993) CSR2, CSR6, CSR26, CSR27, CSR50, PAM114 and PAM117 were performed well in all the three seasons except CSR6 and CSR26 which didn't performed well in summer and autumn whereas, APS4, APS5 and SK6, SK7 performed well in spring, summer and summer, autumn respectively. Based on their performance in all the three seasons, these parental breeds will be utilized for development of foundation crosses which will be further shortlisted for the development of bivoltine silkworm double hybrid suitable to temperate region of J\&K as an alternate for ruling CSR double hybrid.

\section{Introduction}

The common silkworm Bombyx mori Linnaeus (Lepidoptera: Bombycidae) spins valuable silk fibre, making it one of the most beneficial insect to mankind and is becoming an attractive multifunctional material for both textile and non-textile uses Tsukada (2005). The practice of sericulture consist of two major activities viz., cultivation of mulberry plants for producing healthy leaf to the silkworm larvae and the rearing of silkworm larvae to produce the quality cocoons, which is the raw material for the silk reeling industry (Bharath Kumar et al., 2017; Bharath Kumar et al., 2018a). Quality of leaf influences the healthy growth of silkworm larvae and thereby the quality of cocoons (Hajare et al., 2008). With the re-orientation of silkworm breeding approaches aimed at sustainability and 
increased qualitative silk production, unstinted and coordinated efforts by various silkworm breeders in the country (Datta, 1984; Basavaraja et al., 1995 and Ramesh Babu et al., 2002) resulted in the development of many bivoltine silkworm breeds and hybrids over the last few decades. Systematic breeding approaches adapted by various silkworm breeders in different sericulturally advanced countries (Hirobe, 1968; Krisnaswamy and Tikoo, 1971; He yi, 1991; Mano et al., 1991; Hong et al., 1992; Thiagarajan et al., 1993 and Datta et al., 2001) have contributed to produce silkworm (Bombyx mori L.) genotypes of desirable constitution and improvement of several quantitative and qualitative traits of economic value. Till date limited number of silkworm breeds / hybrids has been developed for the spring season under temperate climatic conditions of Kashmir (Trag et al., 1992; Kamili, 1996; Malik et al., 2006 and Malik et al., 2010) Whereas, during summer 10-15 percent farmers take up sericulture in temperate regions of Kashmir (Sahaf et al., 2016) few attempts have also been made for identification of hybrids for summer or autumn season (Farooq et al., 2006; Malik et al., 2009 and Nisar, et al., 2013). It is also need of hour to develop silkworm breeds/ hybrids withstanding the climate change (Bharath Kumar et al., 2018b)

Hence, the present study aims to evaluate the performance of breeds in all the three seasons at CSR\&TI, Pampore to develop the foundation crosses which will be shortlisted for the development of bivoltine silkworm double hybrid suitable for Kashmir valley.

\section{Materials and Methods}

Eleven silkworm genotypes \{CSR2, CSR6, CSR27, CSR26 and CSR50 [CSR\&TI, Mysore] PAM114 and PAM117 [CSR\&TI, Pampore] APS4 and APS5 [APSSRDI, Hindupur] SK6 and SK7 [CSR\&TI,
Berhampore]\} procured from different Institutes were reared during spring (MayJune), summer (July-August) and autumn (August- September), 2017 at Central Sericultural Research and Training Institute, Central Silk Board, Pampore, Kashmir. The characteristics of the parental breeds are presented in Table 1. The standard rearing techniques were followed Krishnaswamy (1978). The important quantitative and qualitative traits viz., fecundity, hatching percentage, yield per 10,000 larvae by weight, single cocoon weight, single shell weight, shell ratio and pupation rate were recorded in all the eleven silkworm breeds during spring, summer and autumn, 2017. All the breeds were reared following completely randomised design with three replications each and 250 larvae were maintained in each replication after $3^{\text {rd }}$ moult. At the end of $5^{\text {th }}$ instar, the spinning larvae were collected manually and mounted in plastic collapsible mountages.

The evaluation index value was calculated for all the eight traits studied. The evaluation index (EI) was calculated as per the below mentioned procedure (Mano et al., 1993).

Evaluation Index $=\frac{\mathrm{A}-\mathrm{B}}{\mathrm{C}}$

Where,

$\mathrm{A}=$ Value obtained for a particular trait in a particular breed

$\mathrm{B}=$ Mean value of a particular trait of all the breeds

$\mathrm{C}=$ Standard deviation of a particular trait of all the breeds

$10=$ Standard unit

$50=$ Fixed value 
The index value obtained for all the traits was combined and the average EI values were obtained. The EI value fixed for the selection of a line is 50 or $>50$. The line, which scored above the limit, is considered to possess greater economic value.

\section{Results and Discussion}

The present research findings revealed that nine breeds viz., CSR2 (50.20), CSR6 (52.31), CSR26 (50.58), CSR27 (50.11), CSR50 (54.31), PAM114 (51.18) PAM117 (55.29), APS4 (54.06) and APS5 (52.68) were performed well in the spring season except SK6 (40.19) and SK7 (38.52). During summer season except CSR6 (45.28) and CSR26 (43.78) remaining breeds viz., CSR2 (51.91), CSR27 (52.31), CSR50 (55.65), PAM114 (51.06) PAM117 (50.04), APS4 (50.13) and APS5 (50.01), SK6 (52.28) and SK7 (52.74) shown E.I above 50.

In autumn CSR2 (50.71), CSR27 (53.46), CSR50 (51.48), PAM114 (59.60) PAM117 (50.14), SK6 (50.31) and SK7 (53.48) recorded E.I above 50 except CSR6 (45.41), CSR26 (41.83), APS4 (44.88) and APS5 (47.20) (Table 8). Since these breeds originated from different parts of the country, the performance of these breeds varies from season to season indicating the degree of variability in genetic potential.

The performance of eleven breeds during spring, summer and autumn 2017 is depicted in Table 2, 4 and 6. CSR breeds from Mysore and PAM breeds from Pampore performed well in all the three seasons except CSR6 and CSR26 which didn't performed well in summer and autumn season. APS breeds from Hindupur performed well in spring and summer only whereas SK breeds from Berhampore recorded E.I value above 50 during summer and autumn, 2017. Evaluation index values of all the parental breeds for particular traits during spring, summer and autumn, 2017 is depicted in Table 3, 5 and 7.

Success of any breeding programme is dependent on the degree of genetic variability of the available genetic resources and the selection of suitable and genetically diverse parental lines to build heterotic combinations. Results of various economic parameters showed significant difference among the breeds which revealed their genetic potential as well as variability of the breeds. These breeds have immense potentiality as breeding material as they displayed adequate variability for economic traits.

Higher the variability more is the scope for obtaining higher amount of heterotic potential and also more variability is expected to surface in segregating generations of such crosses (Shabir Ahmad Bhat et al., 2018). Similar studies based on evaluation index values had also been conducted by Naseema Begum (2000), Quadir et al., (2000), Suresh Kumar et al., (2006), Nazia Choudhary and Ravindra Singh (2006), Ganaie et al., (2012), Nisar et al., (2013) and Nooruldin et al., (2014).

The eleven parental breeds utilised for the present study performed well during Spring (CSR2, CSR6, CSR26, CSR27, CSR50, PAM114, PAM117, APS4 and APS5), summer (CSR2, CSR27, CSR50, PAM114, PAM117, SK6, SK7, APS4 and APS5) and autumn (CSR2, CSR27, CSR50, PAM114, PAM117, SK6 and SK7), 2017.

The variation in the performance of these breeds during three different seasons indicates the degree of genetic variability. The identified parental breeds will be utilised for further breeding programmes to develop foundation crosses which will be further shortlisted to develop bivoltine double hybrid suitable to temperate region of $J \& K$ as an alternate for ruling CSR double hybrid. 
Table.1 Characteristic features of the eleven silkworm parental breeds

\begin{tabular}{|c|c|c|c|c|c|c|c|c|c|c|}
\hline $\begin{array}{l}\text { S. } \\
\text { No. }\end{array}$ & Breeds & $\begin{array}{c}\text { Larval } \\
\text { Marking }\end{array}$ & $\begin{array}{c}\text { Cocoon } \\
\text { Colour }\end{array}$ & $\begin{array}{c}\text { Cocoon } \\
\text { Shape }\end{array}$ & $\begin{array}{c}\text { S. } \\
\text { No. }\end{array}$ & Breeds & $\begin{array}{c}\text { Larval } \\
\text { Marking }\end{array}$ & $\begin{array}{c}\text { Cocoon } \\
\text { Colour }\end{array}$ \\
\hline $\mathbf{1}$ & CSR2 & Plain & White & Oval & 7 & PAM117 & Plain & White & Constricted \\
\hline $\mathbf{2}$ & CSR6 & Marked & White & Constricted & 8 & APS4 & Plain & White & Constricted \\
\hline $\mathbf{3}$ & CSR26 & Marked & White & Constricted & 9 & APS5 & Plain & White & Oval \\
\hline $\mathbf{4}$ & CSR27 & Plain & White & Oval & 10 & SK6 & Plain & White & Constricted \\
\hline $\mathbf{5}$ & CSR50 & Plain & White & Oval & 11 & SK7 & Plain & White & Constricted \\
\hline $\mathbf{6}$ & PAM114 & Plain & White & Oval & & & & & \\
\hline
\end{tabular}

Table.2 Rearing Performance of the eleven silkworm parental breeds during spring, 2017

\begin{tabular}{|c|c|c|c|c|c|c|c|c|c|}
\hline \multirow[t]{2}{*}{ S. No. } & \multirow[t]{2}{*}{$\begin{array}{l}\text { Parental } \\
\text { breeds }\end{array}$} & \multirow[t]{2}{*}{$\begin{array}{l}\text { Fecundity } \\
\text { (No.) }\end{array}$} & \multirow[t]{2}{*}{$\begin{array}{l}\text { Hatching } \\
(\%)\end{array}$} & \multicolumn{2}{|c|}{$\begin{array}{l}\text { Yield / 10, } 000 \\
\text { larvae brushed }\end{array}$} & \multirow[t]{2}{*}{$\begin{array}{c}\text { Single cocoon } \\
\text { wt. (g) }\end{array}$} & \multirow[t]{2}{*}{$\begin{array}{c}\text { Single shell } \\
\text { wt.(g) }\end{array}$} & \multirow[t]{2}{*}{$\begin{array}{c}\text { Shell ratio } \\
(\%)\end{array}$} & \multirow{2}{*}{$\begin{array}{l}\text { Pupation } \\
\text { rate } \\
(\%)\end{array}$} \\
\hline & & & & By No. & By Wt.(kg) & & & & \\
\hline 1 & CSR-2 & 515 & 97.02 & 9500 & 15.04 & 1.60 & 0.32 & 20.18 & 91.33 \\
\hline 2 & CSR-6 & 523 & 96.93 & 9486 & 14.49 & 1.62 & 0.34 & 20.93 & 91.08 \\
\hline 3 & CSR-26 & 510 & 94.41 & 9475 & 14.78 & 1.62 & 0.33 & 20.59 & 91.59 \\
\hline 4 & CSR-27 & 475 & 95.80 & 9501 & 15.00 & 1.64 & 0.34 & 20.48 & 90.59 \\
\hline 5 & CSR-50 & 526 & 95.57 & 9504 & 15.03 & 1.67 & 0.34 & 20.33 & 90.69 \\
\hline 6 & Pam-114 & 500 & 98.80 & 9538 & 15.01 & 1.61 & 0.33 & 19.95 & 90.58 \\
\hline 7 & Pam-117 & 494 & 96.55 & 9485 & 15.35 & 1.60 & 0.34 & 21.33 & 91.02 \\
\hline 8 & APS-4 & 531 & 96.21 & 9495 & 15.12 & 1.61 & 0.33 & 20.61 & 91.25 \\
\hline 9 & APS-5 & 529 & 97.03 & 9426 & 15.15 & 1.61 & 0.33 & 20.46 & 91.35 \\
\hline 10 & SK-6 & 545 & 97.59 & 9359 & 14.68 & 1.58 & 0.31 & 19.62 & 90.25 \\
\hline \multirow[t]{3}{*}{11} & SK-7 & 460 & 93.12 & 9479 & 14.86 & 1.59 & 0.31 & 19.81 & 90.38 \\
\hline & Avg. & 510 & 96.28 & 9477 & 14.96 & 1.61 & 0.33 & 20.39 & 90.92 \\
\hline & SD & 26 & 1.55 & 47 & 0.24 & 0.03 & 0.01 & 0.49 & 0.44 \\
\hline
\end{tabular}


Table.3 Evaluation index values of eleven silkworm parental breeds during spring, 2017

\begin{tabular}{|c|c|c|c|c|c|c|c|c|c|c|}
\hline \multirow[t]{2}{*}{ S. No. } & \multirow[t]{2}{*}{$\begin{array}{l}\text { Parental } \\
\text { breeds }\end{array}$} & \multirow[t]{2}{*}{$\begin{array}{l}\text { Fecundity } \\
\text { (No.) }\end{array}$} & \multirow[t]{2}{*}{$\begin{array}{c}\text { Hatching } \\
(\%)\end{array}$} & \multicolumn{2}{|c|}{$\begin{array}{l}\text { Yield / 10, } 000 \\
\text { larvae brushed }\end{array}$} & \multirow{2}{*}{$\begin{array}{l}\text { Single } \\
\text { cocoon } \\
\text { wt. (g) }\end{array}$} & \multirow{2}{*}{$\begin{array}{c}\text { Single shell } \\
\text { wt. } \\
\text { (g) }\end{array}$} & \multirow[t]{2}{*}{$\begin{array}{c}\text { Shell ratio } \\
(\%)\end{array}$} & \multirow[t]{2}{*}{$\begin{array}{c}\text { Pupation rate } \\
(\%)\end{array}$} & \multirow[t]{2}{*}{ Mean E.I } \\
\hline & & & & By No. & By Wt.(kg) & & & & & \\
\hline 1 & CSR-2 & 51.92 & 54.77 & 54.89 & 53.33 & 46.67 & 35.00 & 45.71 & 59.32 & 50.20 \\
\hline 2 & CSR-6 & 55.00 & 54.19 & 51.91 & 30.42 & 53.33 & 59.00 & 61.02 & 53.64 & 52.31 \\
\hline 3 & CSR-26 & 50.00 & 37.94 & 49.57 & 42.50 & 52.33 & 53.00 & 54.08 & 65.23 & 50.58 \\
\hline 4 & CSR-27 & 36.54 & 46.90 & 55.11 & 51.67 & 60.33 & 56.00 & 51.84 & 42.50 & 50.11 \\
\hline 5 & CSR-50 & 56.15 & 45.42 & 55.74 & 52.92 & 70.67 & 60.00 & 48.78 & 44.77 & 54.31 \\
\hline 6 & Pam-114 & 46.15 & 66.26 & 62.98 & 52.08 & 48.67 & 50.00 & 41.02 & 42.27 & 51.18 \\
\hline 7 & Pam-117 & 43.85 & 51.74 & 51.70 & 66.25 & 46.33 & 61.00 & 69.18 & 52.27 & 55.29 \\
\hline 8 & APS-4 & 58.08 & 49.55 & 53.83 & 56.67 & 50.33 & 52.00 & 54.49 & 57.50 & 54.06 \\
\hline 9 & APS-5 & 57.31 & 54.84 & 39.15 & 57.92 & 51.00 & 50.00 & 51.43 & 59.77 & 52.68 \\
\hline 10 & SK-6 & 63.46 & 58.45 & 24.89 & 38.33 & 38.33 & 29.00 & 34.29 & 34.77 & 40.19 \\
\hline 11 & SK-7 & 30.77 & 29.61 & 50.43 & 45.83 & 41.67 & 34.00 & 38.16 & 37.73 & 38.52 \\
\hline
\end{tabular}

Table.4 Rearing Performance of the eleven silkworm parental breeds during summer, 2017

\begin{tabular}{|c|c|c|c|c|c|c|c|c|c|}
\hline \multirow[t]{2}{*}{ S. No. } & \multirow[t]{2}{*}{ Parental breeds } & \multirow[t]{2}{*}{$\begin{array}{l}\text { Fecundity } \\
\text { (No.) }\end{array}$} & \multirow[t]{2}{*}{$\begin{array}{c}\text { Hatching } \\
(\%)\end{array}$} & \multicolumn{2}{|c|}{$\begin{array}{c}\text { Yield / 10, } 000 \\
\text { larvae brushed }\end{array}$} & \multirow[t]{2}{*}{$\begin{array}{c}\text { Single cocoon } \\
\text { wt. (g) }\end{array}$} & \multirow[t]{2}{*}{$\begin{array}{c}\text { Single shell } \\
\text { wt.(g) }\end{array}$} & \multirow[t]{2}{*}{$\begin{array}{c}\text { Shell ratio } \\
(\%)\end{array}$} & \multirow[t]{2}{*}{$\begin{array}{c}\text { Pupation rate } \\
(\%)\end{array}$} \\
\hline & & & & By No. & By Wt.(kg) & & & & \\
\hline 1 & CSR-2 & 524 & 98.09 & 9693 & 15.08 & 1.59 & 0.32 & 20.06 & 90.50 \\
\hline 2 & CSR-6 & 507 & 98.18 & 9507 & 14.63 & 1.58 & 0.32 & 20.51 & 90.45 \\
\hline 3 & CSR-26 & 506 & 97.87 & 9600 & 14.71 & 1.57 & 0.31 & 20.04 & 90.51 \\
\hline 4 & CSR-27 & 507 & 97.94 & 9667 & 14.89 & 1.58 & 0.33 & 21.05 & 90.00 \\
\hline 5 & CSR-50 & 538 & 98.85 & 9693 & 14.89 & 1.59 & 0.33 & 20.60 & 90.48 \\
\hline 6 & Pam-114 & 556 & 97.45 & 9640 & 14.81 & 1.58 & 0.32 & 20.56 & 90.29 \\
\hline 7 & Pam-117 & 544 & 96.80 & 9612 & 14.59 & 1.57 & 0.33 & 20.83 & 91.00 \\
\hline 8 & APS-4 & 557 & 96.71 & 9667 & 14.65 & 1.55 & 0.32 & 20.65 & 91.29 \\
\hline 9 & APS-5 & 542 & 97.29 & 9640 & 14.95 & 1.59 & 0.32 & 20.12 & 90.48 \\
\hline 10 & SK-6 & 516 & 96.92 & 9600 & 15.09 & 1.61 & 0.33 & 20.24 & 91.45 \\
\hline \multirow[t]{3}{*}{11} & SK-7 & 518 & 98.83 & 9667 & 15.16 & 1.60 & 0.32 & 20.09 & 90.05 \\
\hline & Avg. & 529 & 97.72 & 9635 & 14.86 & 1.58 & 0.32 & 20.43 & 90.59 \\
\hline & SD & 19 & 0.75 & 54 & 0.20 & 0.02 & 0.01 & 0.34 & 0.47 \\
\hline
\end{tabular}


Table.5 Evaluation index values of eleven silkworm parental breeds during summer, 2017

\begin{tabular}{|c|c|c|c|c|c|c|c|c|c|c|}
\hline \multirow[t]{2}{*}{ S. No. } & \multirow[t]{2}{*}{$\begin{array}{l}\text { Parental } \\
\text { breeds }\end{array}$} & \multirow[t]{2}{*}{$\begin{array}{l}\text { Fecundity } \\
\text { (No.) }\end{array}$} & \multirow[t]{2}{*}{$\begin{array}{c}\text { Hatching } \\
(\%)\end{array}$} & \multicolumn{2}{|c|}{$\begin{array}{l}\text { Yield / 10, } 000 \\
\text { larvae brushed }\end{array}$} & \multirow{2}{*}{$\begin{array}{l}\text { Single } \\
\text { cocoon } \\
\text { wt. (g) }\end{array}$} & \multirow{2}{*}{$\begin{array}{c}\text { Single shell } \\
\text { wt. } \\
\text { (g) }\end{array}$} & \multirow[t]{2}{*}{$\begin{array}{c}\text { Shell ratio } \\
(\%)\end{array}$} & \multirow[t]{2}{*}{$\begin{array}{c}\text { Pupation rate } \\
(\%)\end{array}$} & \multirow[t]{2}{*}{ Mean E.I } \\
\hline & & & & By No. & By Wt.(kg) & & & & & \\
\hline 1 & CSR-2 & 47.37 & 54.93 & 60.74 & 61.00 & 55.00 & 49.00 & 39.12 & 48.09 & 51.91 \\
\hline 2 & CSR-6 & 38.42 & 56.13 & 26.30 & 38.50 & 49.50 & 54.00 & 52.35 & 47.02 & 45.28 \\
\hline 3 & CSR-26 & 37.89 & 52.00 & 43.52 & 42.50 & 43.50 & 44.00 & 38.53 & 48.30 & 43.78 \\
\hline 4 & CSR-27 & 38.42 & 52.93 & 55.93 & 51.50 & 51.00 & 63.00 & 68.24 & 37.45 & 52.31 \\
\hline 5 & CSR-50 & 54.74 & 65.07 & 60.74 & 51.50 & 53.50 & 57.00 & 55.00 & 47.66 & 55.65 \\
\hline 6 & Pam-114 & 64.21 & 46.40 & 50.93 & 47.50 & 48.00 & 54.00 & 53.82 & 43.62 & 51.06 \\
\hline 7 & Pam-117 & 57.89 & 37.73 & 45.74 & 36.50 & 45.00 & 57.00 & 61.76 & 58.72 & 50.04 \\
\hline 8 & APS-4 & 64.74 & 36.53 & 55.93 & 39.50 & 35.00 & 48.00 & 56.47 & 64.89 & 50.13 \\
\hline 9 & APS-5 & 56.84 & 44.27 & 50.93 & 54.50 & 55.00 & 50.00 & 40.88 & 47.66 & 50.01 \\
\hline 10 & SK-6 & 43.16 & 39.33 & 43.52 & 61.50 & 63.00 & 55.00 & 44.41 & 68.30 & 52.28 \\
\hline 11 & SK-7 & 44.21 & 64.80 & 55.93 & 65.00 & 61.50 & 52.00 & 40.00 & 38.51 & 52.74 \\
\hline
\end{tabular}

Table.6 Rearing Performance of the eleven silkworm parental breeds during autumn, 2017

\begin{tabular}{|c|c|c|c|c|c|c|c|c|c|}
\hline \multirow[t]{2}{*}{ S. No. } & \multirow[t]{2}{*}{ Parental breeds } & \multirow[t]{2}{*}{$\begin{array}{l}\text { Fecundity } \\
\text { (No.) }\end{array}$} & \multirow[t]{2}{*}{$\begin{array}{l}\text { Hatching } \\
(\%)\end{array}$} & \multicolumn{2}{|c|}{$\begin{array}{l}\text { Yield / 10, } 000 \\
\text { larvae brushed }\end{array}$} & \multirow[t]{2}{*}{$\begin{array}{l}\text { Single cocoon } \\
\text { wt. (g) }\end{array}$} & \multirow[t]{2}{*}{$\begin{array}{l}\text { Single shell } \\
\text { wt.(g) }\end{array}$} & \multirow[t]{2}{*}{$\begin{array}{c}\text { Shell ratio } \\
(\%)\end{array}$} & \multirow[t]{2}{*}{$\begin{array}{c}\text { Pupation rate } \\
(\%)\end{array}$} \\
\hline & & & & By No. & By Wt.(kg) & & & & \\
\hline 1 & CSR-2 & 528 & 98.65 & 9533 & 14.61 & 1.569 & 0.308 & 19.63 & 91.58 \\
\hline 2 & CSR-6 & 518 & 98.26 & 9507 & 14.44 & 1.552 & 0.307 & 19.78 & 91.44 \\
\hline 3 & CSR-26 & 518 & 98.17 & 9507 & 14.37 & 1.547 & 0.302 & 19.52 & 91.48 \\
\hline 4 & CSR-27 & 521 & 98.08 & 9653 & 14.73 & 1.562 & 0.314 & 20.1 & 91.42 \\
\hline 5 & CSR-50 & 535 & 98.69 & 9653 & 14.8 & 1.568 & 0.306 & 19.52 & 91.14 \\
\hline 6 & Pam-114 & 539 & 98.9 & 9693 & 14.75 & 1.557 & 0.316 & 20.3 & 91.32 \\
\hline 7 & Pam-117 & 540 & 98.6 & 9615 & 14.43 & 1.55 & 0.311 & 20.06 & 90.89 \\
\hline 8 & APS-4 & 557 & 97.94 & 9520 & 14.2 & 1.518 & 0.308 & 20.29 & 90.99 \\
\hline 9 & APS-5 & 545 & 97.92 & 9507 & 14.47 & 1.56 & 0.307 & 19.68 & 91.47 \\
\hline 10 & SK-6 & 515 & 98.14 & 9462 & 14.51 & 1.586 & 0.313 & 19.84 & 91.78 \\
\hline \multirow[t]{3}{*}{11} & SK-7 & 528 & 98.72 & 9547 & 14.75 & 1.586 & 0.313 & 19.74 & 91.22 \\
\hline & Avg. & 531 & 98.37 & 9563 & 14.55 & 1.56 & 0.31 & 19.86 & 91.34 \\
\hline & $\mathrm{SD}$ & 13 & 0.35 & 76 & 0.19 & 0.02 & 0.004 & 0.29 & 0.26 \\
\hline
\end{tabular}


Table.7 Evaluation index values of eleven silkworm parental breeds during autumn, 2017

\begin{tabular}{|c|c|c|c|c|c|c|c|c|c|c|}
\hline \multirow[t]{2}{*}{ S. No. } & \multirow[t]{2}{*}{$\begin{array}{l}\text { Parental } \\
\text { breeds }\end{array}$} & \multirow[t]{2}{*}{$\begin{array}{l}\text { Fecundity } \\
\text { (No.) }\end{array}$} & \multirow[t]{2}{*}{$\begin{array}{c}\text { Hatching } \\
(\%)\end{array}$} & \multicolumn{2}{|c|}{$\begin{array}{l}\text { Yield / 10, } 000 \\
\text { larvae brushed }\end{array}$} & \multirow{2}{*}{$\begin{array}{l}\text { Single } \\
\text { cocoon } \\
\text { wt. (g) }\end{array}$} & \multirow{2}{*}{$\begin{array}{l}\text { Single } \\
\text { shell wt. } \\
\text { (g) }\end{array}$} & \multirow[t]{2}{*}{$\begin{array}{c}\text { Shell ratio } \\
(\%)\end{array}$} & \multirow{2}{*}{$\begin{array}{c}\text { Pupation } \\
\text { rate } \\
(\%)\end{array}$} & \multirow[t]{2}{*}{ Mean E.I } \\
\hline & & & & By No. & By Wt.(kg) & & & & & \\
\hline 1 & CSR-2 & 47.69 & 58.00 & 46.05 & 53.16 & 54.50 & 45.00 & 42.07 & 59.23 & 50.71 \\
\hline 2 & CSR-6 & 40.00 & 46.86 & 42.63 & 44.21 & 46.00 & 42.50 & 47.24 & 53.85 & 45.41 \\
\hline 3 & CSR-26 & 40.00 & 44.29 & 42.63 & 40.53 & 43.50 & 30.00 & 38.28 & 55.38 & 41.83 \\
\hline 4 & CSR-27 & 42.31 & 41.71 & 61.84 & 59.47 & 51.00 & 60.00 & 58.28 & 53.08 & 53.46 \\
\hline 5 & CSR-50 & 53.08 & 59.14 & 61.84 & 63.16 & 54.00 & 40.00 & 38.28 & 42.31 & 51.48 \\
\hline 6 & Pam-114 & 56.15 & 65.14 & 67.11 & 60.53 & 48.50 & 65.00 & 65.17 & 49.23 & 59.60 \\
\hline 7 & Pam-117 & 56.92 & 56.57 & 56.84 & 43.68 & 45.00 & 52.50 & 56.90 & 32.69 & 50.14 \\
\hline 8 & APS-4 & 70.00 & 37.71 & 44.34 & 31.58 & 29.00 & 45.00 & 64.83 & 36.54 & 44.88 \\
\hline 9 & APS-5 & 60.77 & 37.14 & 42.63 & 45.79 & 50.00 & 42.50 & 43.79 & 55.00 & 47.20 \\
\hline 10 & SK-6 & 37.69 & 43.43 & 36.71 & 47.89 & 63.00 & 57.50 & 49.31 & 66.92 & 50.31 \\
\hline 11 & SK-7 & 47.69 & 60.00 & 47.89 & 60.53 & 63.00 & 57.50 & 45.86 & 45.38 & 53.48 \\
\hline
\end{tabular}

Table.8 Mean Evaluation index values of eleven silkworm parental breeds during spring, summer and autumn 2017

\begin{tabular}{|c|c|c|c|c|c|c|c|c|c|c|c|c|}
\hline $\begin{array}{c}\text { Breeds } \\
\text { Seasons }\end{array}$ & CSR-2 & CSR-6 & CSR-26 & CSR-27 & CSR-50 & Pam-114 & Pam-117 & APS-4 & APS-5 & SK-6 & SK-7 \\
\hline Spring & 50.20 & 52.31 & 50.58 & 50.11 & 54.31 & 51.18 & 55.29 & 54.06 & 52.68 & 40.19 & 38.52 \\
\hline Summer & 51.91 & 45.28 & 43.78 & 52.31 & 55.65 & 51.06 & 50.04 & 50.13 & 50.01 & 52.28 & 52.74 \\
\hline Autumn & 50.71 & 45.41 & 41.83 & 53.46 & 51.48 & 59.60 & 50.14 & 44.88 & 47.20 & 50.31 & 53.48 \\
\hline
\end{tabular}




\section{Acknowledgments}

Authors wish to express a deep sense of gratitude to Central Silk Board and Central Sericultural Research and Training Institute, Pampore, Jammu and Kashmir for providing financial assistance and laboratory facilities to carry out the research work.

\section{References}

Basavaraja, H. K., Nirmal Kumar, S., Suresh Kumar, N., Mal Reddy, N., Kshama Giridhar, Ahshan, M. M. and Datta, R. K. (1995). New productive bivoltine hybrids. Indian Silk, 34: 5 - 9.

Bharath Kumar Neelaboina., Gulzar Ahmad Khan, Mudasir Gani, Shakeel Ahmad, Mir Nisar Ahmad and M.K. Ghosh (2018a). Exploration of sericulture in unexplored region of Jammu and Kashmir. Journal of Entomol. Zool. Studies, 6(4): 1922-1925.

Bharath Kumar Neelaboina., Shivkumar, Gulzar Ahmad khan, Mudasir Gani, Mir Nisar Ahmad and M.K. Ghosh (2018b). Impact of Climate Change on Agriculture and allied sectors. Journal of Entomolozy and Zoolozy Studies, 6(5): 426-429

Bharath Kumar, Neelaboina, Shivkumar, Gani, M., Babulal and Ghosh, M. K. (2017). Assessment of performance of autumn crop over spring in temperate region of Jammu \& Kashmir. Journal of Agroecology and Natural Resource Management, 4 (2): 112 - 114.

Datta, R. K., Basavaraja, H. K., Mal Reddy, N., Nirmal Kumar, S., Suresh Kumar, N. Ramesh Babu, M. Ahsan, M.M. and Jayaswal, K.P. (2001). Breeding of new productive bivoltine hybrid, CSR12 $\mathrm{x}$ CSR6 of silkworm, Bombyx mori L. Int. J. Indust. Entomol, 3: 127 - 133.
Datta, R.K. (1984). Improvement of silkworm race (Bombyx mori L.) in India. Sericologia, 24: 393 - 415.

Farooq M, Singh TP, Nooruddin, Rufaie Z. H., Baqual M., Dar H. U. (2006) Second commercial crop to make sericulture a more profitable in Kashmir. Proceedings of regional Seminar on Prospects and Problems of Sericulture as are economic enterprise in North West India. 275-276.

Ganie, N. A., Kamili, A.S., Baqual, M. F., Sharma, R.K., Dar, K.A. and Khan, I.L. (2012). Indian sericulture industry with particular reference to Jammu \& Kashmir. Intl. J. Appld. Biol. Res, 2(2): 194-202.

Hajare, T. N., Jadhav, A. D., Jagdish Prasad., Patil, N. G and Lal, S. (2008). Performance of silkworm breeds (Bombyx mori L.) in Vidarbha region during summer. Indian Journal of Sericulture, 47 (1): 111-114.

He, Y.I., Sima, Yang-lu., Jiang Da-xin. and Dai, ping. (1991). Breeding of the silkworm varieties for summer and autumn rearing, "Xuhua", "Qiuxing" and their hybrids. Acta Sericologia Sinica, 17(4): 200-207.

Hirobe, T. (1968). Evolution, differentiation and breeding of the silkworm. The Silk Road past and present- genetics in Asian countries. In: XII Intl. Congr. Genetics, pp. 25-36.

Hong, K.W., Hwang, S.J., Ryu, K.S., Choi, S.R., Kim, K.Y. and Lee, S.P. (1992). Breeding of Bunongjam, a high silk yielding silkworm variety for spring rearing season. Research Reports of the Rural Development Administration, Farm Management, Agricultural Engineering, Sericulture and Farm Products Utilization, 34(1): 30-35.

Kamili AS. (1996). New bivoltine silkworm breeds and their hybrids (SKAU-HR-1) Technical Document. Sher-e-Kashmir 
University of Agricultural Sciences and Technology of Kashmir (J \& K),

Krishnaswami, S. (1978). New technology of silkworm rearing, Bulletin No. 2, CSR\&TI, Mysore, India, pp. 1-24.

Krishnaswami, S. and Tikoo, B. L. (1971). A Comparative study of performance of pure races currently under rearing in Mysore State. Indian J. Seric, 10: 66 71.

Malik GN, Rufaie SZ, Baqual MF, Kamilli AS, Dar HU. (2006). Comparative performance of some bivoltine silkworm, Bombyx mori L. hybrids. Entomon, 1:61-64.

Malik MA, Kamili AS, Sofi AM, Malik GN, Sabahat A, Bhat SA. Second commercial silkworm rearing in Kashmir - A ray of hope. Indian Silk, 9:10-11.

Malik MA, Kamilli AS, Sofi AM, Malik GN, Sabahat A, Malik FA. (2010). Evaluation and identification of region / season specific bivoltine hybrids of the silkworm, Bombyx mori L. suitable for Kashmir climatic conditions. Journal of Experimental Zoology India. 13:171176.

Mano, Y., Nirmalkumar, S., Basavaraja, H.K., Mal Reddy, N. and Datta, R.K. (1993). A new method to select promising silkworm breed/hybrid combinations. Indian Silk, 31(10): 53.

Mano, Y., Ohyanagi, M., Nagayasu, K. and Murakami, A. (1991). Breeding of sexlimited larval marking silkworm [Bombyx mori] race, N147 x C145. Bull. Natio. Inst. Seril. Entomol. Scie, 2: 1-29.

Naseema Begum, A., Basavaraja, H.K., Sudhakara Rao, P., Rekha, M. and Ahsan, M.M. (2000). Identification of bivoltine silkworm hybrids suitable for tropical climate. Indian. J. Seric, 39(1): 24-29.
Nazia Choudhary and Ravindra Singh. (2006). Evaluation of few polyvoltine $x$ bivoltine hybrids of the silkworm Bombyx mori L. Indian. J. Seric, 45(1): 62-65.

Nisar, M., Chisti, M.Z. and Khan, M.A. (2013). Studies on the identification of summer specific silkworm Bombyx mori L. hybrids under temperate climatic conditions of Jammu and Kashmir, India. J. Intl. Acad. Res. Multidisci, 1(3): 1-14

Nooruldin, S., Bhat, S. A., Malik, M. A., Khan, I. L. and Sahaf, K. A. (2014). Comparative performance of silkworm, Bombyx mori L. hybrids during different seasons under Kashmir climatic conditions. Green Farming, 6(6): 1392-1395.

Quadir, S. M., Nisar, M., Khan, M. A. and Ahsan, M. M. (2000). Identification of season specific silkworm hybrids for temperate climatic conditions of Kashmir. In: National Conference on Strategies for Sericulture Research and Development, Central Sericultural Research and Training Institute, Srirampura, Mysore, India, 16-18, November 2000, pp.21.

Ramesh Babu, M., Chandrashekaraiah, Lakshmi, H. and Prasad, J. (2002). Multiple trait evaluation of bivoltine hybrids of silkworm, Bombyx mori (L.). Int. J. Indust. Entomol, 5(1): 37-43.

Sahaf KA, Bhat SA, Mir Nisar A. (2016). Sericulture in North-west India with special reference to temperate regionproblems and prospects. National seminar on sericulture development in temperate region- problems and prospects. $34-38$

Shabir Ahmad Bhat, Ravi Kant, Naveena Nazim, Mohd Rafiq Bhat, Noor-ul-Din, Munazah Yaqoob and Nageena Nazir (2018). Genetic variability analysis of some bivoltine silkworm (Bombyx mori 
L.) breeds. Journal of Pharmacognosy and Phytochemistry, 7(3): 889-892

Suresh Kumar, N., Basavaraja, H.K., Joge, P.G., Mal Reddy, N., Kalpana, G.V. and Dandin, S.B. (2006). Development of new robust bivoltine hybrid (CSR46 x CSR47) of Bombyx mori L. for the tropics. Indian J. Seric, 45(1): 21-29.

Thiagarajan, V., Bhargava, S. K., Ramesh Babu, M. and Nagaraj, B. (1993). Difference in seasonal performance of
26 strains of silkworm Bombyx mori. (Bombycidae). J. Lep. Soc, 47(4): 321337.

Trag AR, Kamili AS, Malik GN, Kukiloo FA. (1992). Evolution of high yielding bivoltine silkworm, Bombyx mori genotypes. Sericologia. 32:321-324.

Tsukada, M., Islam, S., Arai, T., Bosch, A and Fred, G. (2005). Microwave irradiation technique to enhance protein fiber properties. Autex Res J, 5(1): 40-8.

\section{How to cite this article:}

Bharath Kumar Neelaboina, Shivkumar, Mir Nisar Ahmad and Ghosh, M.K. 2018. Studies on the Performance of Some Silkworm, Bombyx mori L, Breeds in Temperate Region of Jammu and Kashmir, India. Int.J.Curr.Microbiol.App.Sci. 7(11): 2192-2201. doi: https://doi.org/10.20546/ijcmas.2018.711.245 